\title{
UHF-RFID Desktop Reader Antennas: Performance Analysis in the Near-Field Region
}

\author{
Andrea Michel, Student Member, IEEE, and Paolo Nepa, Member, IEEE
}

\begin{abstract}
In this letter, a metric is proposed to qualitatively predict the achievable reading performance of a near-field (NF) UHFRFID (865-928 MHz) desktop reader antenna, through a numerical analysis. Specifically, since the typical far-field antenna parameters (gain, radiation pattern, axial ratio) are not effective in the near-field region, the normalized power density is here proposed as a near-field antenna performance parameter. For a given NF antenna, the mean value of the normalized power density is evaluated on an area parallel to the antenna surface, at a set of different distances. Then, its decay rate is studied as a function of the distance from the antenna surface, which is useful to compare reading range performance of different antenna layouts. Additionally, the probability density function of the normalized power density at a given distance from the antenna surface is considered to investigate the field uniformity characteristics, the latter being important to guarantee tag reading independently on its location with respect to the reader antenna. The proposed analysis is applied to a set of NF UHF-RFID printed antennas, and conclusions are validated through a set of experimental tests.
\end{abstract}

Index Terms-Near-field antenna characterization, near-field coupling, near-field UHF-RFID systems, UHF-RFID reader antenna.

\section{INTRODUCTION}

Near-field radio frequency identification (NF-RFID) systems are being widely used for item-level tagging in pharmaceutical and retailing industries [1]. Since in NF applications the tag detection has to be limited within an assigned confined volume close to the antenna surface, a proper reader antenna design is required. A number of solutions for NF UHF- RFID reader antennas have been proposed, such as arrays of patches [2], strips [2], or slots [4] at UHF and microwaves (e.g., $2.4 \mathrm{GHz}$ ) frequency bands. Segmented loop antennas [5]-[8] have been proposed to get an almost constant current distribution in a physically large loop, where contiguous loop segments are connected by lumped [5] or distributed [6]-[8] capacitive elements. More recently, traveling wave antennas (TWAs) have been proposed for NF UHF-RFID systems [8]-[13]. Since the length and shape of the radiating transmission line are not strictly related to the operating frequency (non-resonating structures), TWAs can be changed in size quite easily to be com- pliant with the specifications on the reader interrogation volume.

In this context, microstrip line technology has been often ap- plied [8]-[10]. A straight coplanar stripline (CPS) is also proposed in [11]. In [12] and [13], the authors proposed a single meander line and a meandered $2 \times 2$ array based on coplanar waveguide (CPW) technology, respectively. Furthermore, to extend tag readability up to a few decimeters from the antenna surface, a modular antenna concept has been introduced by the authors in [14] and [15]. The modular antenna comprises a TWA and a low-gain resonating antenna, which are combined to meet specific requirements on the spatial decay rate and amplitude of the radiated field, in the reactive and radiative antenna near-field regions.

Typical far-field antenna parameters are not effective to fully

characterize antennas for NF applications, and they are independent on the distance from the antenna. On the other hand, any metric for NF antenna characterization is strictly related to the distance from the antenna surface. Moreover, in the NF region, the radial field component may be nonnegligible, and in some designs, it is even maximized to increase the inductive coupling when using loop-like tags parallel to the antenna sur- face (e.g., [11]). In this context, an antenna parameter that can be calculated from reliable numerical simulations of the antenna near field is looked for, which also relates to the reader detection performance.

In this letter, the power density (amplitude of the real component of the Poynting vector) is proposed as a parameter to qualitatively predict the achievable reading performance of a desktop reader antenna, through a numerical analysis of both the electric and magnetic vector fields in a selected volume close to the reader antenna. In Section II, this parameter is discussed, and simulated results are shown for a set of desktop reader antennas already presented in the scientific literature. Specifically, the variation of the power density mean value versus the distance from the antenna surface provides a qualitative estimation of the field amplitude decay rate, which relates to the reader reading range performance. Moreover, the probability density function of the power density allows to estimate the field distribution uniformity in a specific area, which relates to the probability of missed reading events due to the presence of low-field regions on the antenna surface. It is underlined that this research is not aimed to characterize a complete near-field RFID system, but it is mainly focused to propose a metric to compare different reader antenna solutions, without any constraint on the tag typology, scenario, or reader settings. Then, in Section III, tag detection measurements with a commercial UHF-RFID setup are used to show the effectiveness of the power density numerical analysis in a qualitative estimate of the performance of an NF UHF-RFID reader antenna. 
II.

Let us consider a typical layout of an antenna suitable for UHF-RFID desktop readers, which consists of a low-profile planar antenna with a size of the order of $\lambda \times \lambda$ or slightly less ( $\lambda$ being the free-space wavelength at UHF-RFID band). Then, if the tag is supposed to lie at a distance from the antenrałsur- face less than $10-15 \mathrm{~cm}$, it is apparent that the field-observation points are in the antenna NF region. In this region, all the field components are excited. In particular, the magnetic field could be reasonably considered as the key-parameter to be maximized in order to improve the detection of loop- or spiral-like tags (inductive coupling), as in [10] and [11]. Similarly, in [2], [4], and [9], the electric field distribution is taken into account since it is related to the detection of dipole-like tags (capacitive coupling). Despite this, several commercial UHF RFID tags are characterized by a hybrid layout, and they cannot be considered electrically small (i.e., their size is not lower than $\lambda / 10$ ). For these tags, the electric and magnetic energy densities can be comparable, and both the electric and magnetic fields are responsible for the tag coupling. In order to take into account the NF characteristics of both electric and magnetic fields radiated by an NF reader antenna, the normalized power density (NPD) is here proposed as a possible metric for comparing different reader antenna layouts

$$
\operatorname{NPD}(\mathrm{x}, \mathrm{y}, \mathrm{z})=\frac{\frac{1}{2}\left\|\Re e\left\{\mathbf{E}(\mathrm{x}, \mathrm{y}, \mathrm{z}) \times \mathbf{H}(\mathrm{x}, \mathrm{y}, \mathrm{z})^{*}\right\}\right\|}{\mathrm{P}_{\mathrm{IN}} / \mathrm{A}_{\mathrm{GEOM}}} .
$$

In (1), $\mathbf{E}$ and $\mathbf{H}$ and are the electric and magnetic vector fields, respectively, $\mathrm{P}_{\mathrm{IN}}$ is the antenna input power, and $\mathrm{A}_{\mathrm{GEOM}}$ is the geometrical area of the reader antenna surface. The numerator represents the Euclidean norm of the real component of the Poynting vector. On the other hand, the denominator represents the ratio between the antenna input power and its geometrical area. Specifically, the denominator represents the power density on the antenna surface in the ideal scenario in which the antenna is able to uniformly spread the input power over its entire surface. In a sense, the normalized power density can be thought as an extension of the far-field antenna gain parameter to antennas for NF applications, and it is suitable to compare antennas exhibiting different size. Finally, all the field components are included in (1), for both $\mathbf{E}$ and $\mathbf{H}$.

By way of example, the numerical analysis of the NPD has been carried out on three antenna layouts proposed in the literature [12]-[14]. In particular, the antenna presented in [12] consists of a single-line meandered TWA based on the CPW technologydue to its layout, it has been named snake antenna. Then, the antenna described in [13] consists of a $2 \times 2$ array of meandered TWAs properly fed to confine the field in proximity of the reader antenna central area. Finally, the modular antenna proposed in [14] consists of a spiral microstrip line that serially feeds an array of two miniaturized circularly polarized patch antennas. As a reference, a standard circularly polarized (CP) resønखrle patch antenna has also been considered. All the antennas have been simulated by using CST Microwave Studio. The electric and magnetic vector fields have been computed at various distances from the antenna surface (up to $20 \mathrm{~cm}$ ). The distance

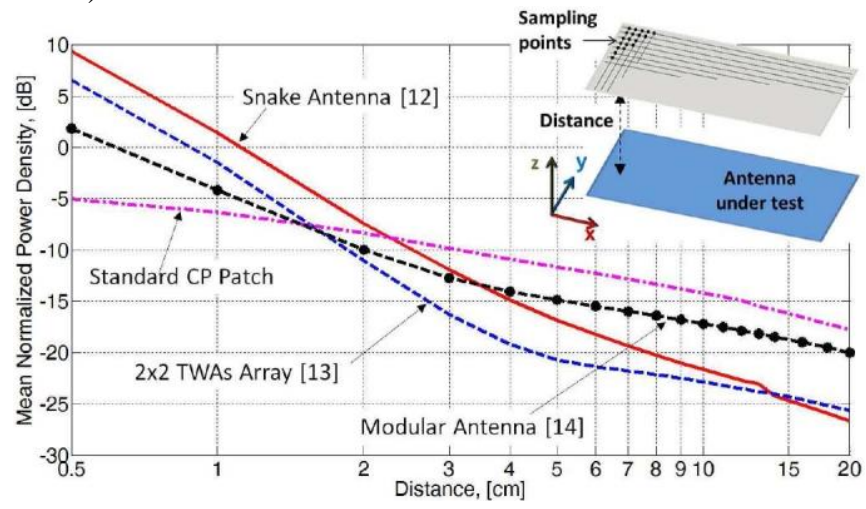

Fig. 1. Mean normalized power density, versus the distance from the antenna surface, for all the antenna configurations taken into account. The normalized power density has been averaged on an area of $275 \times 136 \mathrm{~mm}^{2}$ for [12]-[14] and 160x160 mm for the CP patch, and on a set of frequencies between 902 and $928 \mathrm{MHz}$ (with a 2-MHz frequency step).

between the observation points of the grid has been chosen equal to $1 \mathrm{~mm}$. Thus, both the electric and magnetic vector fields have been processed to evaluate the NPD at each point [(1)]. To take into account for the fact that frequency hopping is implemented in RFID readers to specifically overcome interference issues, the NPD values have been computed in the frequency range between $902-928 \mathrm{MHz}$, with a 2-MHz step. For the three near-field antenna configurations [12]-[14], the 
mean value of the NPD has been computed by averaging the values computed at different frequencies in a selected area $\left(\mathrm{A}_{\mathrm{GEOM}}\right)$ that coincides with the reader antenna size $\left(275 \times 135 \mathrm{~mm}^{2}\right)$, for a set of distances from the antenna surface. For the CP resonating square patch, a 160x $160 \mathrm{~mm}^{2}$ square region has been considered, which corresponds to the ground plane size. In Fig. 1, the mean NPD is plotted as a function of the distance.

As expected, for the snake antenna [12] and the TWAs array [13], the mean NPD rapidly decreases when increasing the distance from the antenna surface - the curve slope is almost $30 \mathrm{~dB} / \mathrm{decade}$. That is, the radiated field is maximized close to the antenna surface, but it exhibits a sharp reduction when moving far from the antenna surface. On the other hand, the curve for CP patch antenna shows a slower amplitude decrease with respect to the snake antenna and TWAs array, so allowing for the detection of bulks of tags. However, close to the reader surface, the mean NPD is significantly lower. The modular antenna offers a multifunction feature to the RFID reader system. Specifically, it allows for a relatively high NPD close to the reader surface, but it decays slower than for the snake antenna and TWAs array antennas, so exhibiting a relatively high field amplitude even in the antenna radiative near-field region.

Antennas for NF UHF-RFID readers have to exhibit a field distribution as uniform as possible on the entire interrogation area (i.e., the reader antenna surface) to avoid missed readings (false negatives). The field distribution uniformity level can be evaluated by considering the probability density function (PDF) of the NPD. Specifically, by calculating the NPD at a set of test points on a geometrical surface at an assigned distance from the antenna surface, a PDF curve can be generated. In Fig. 2, the

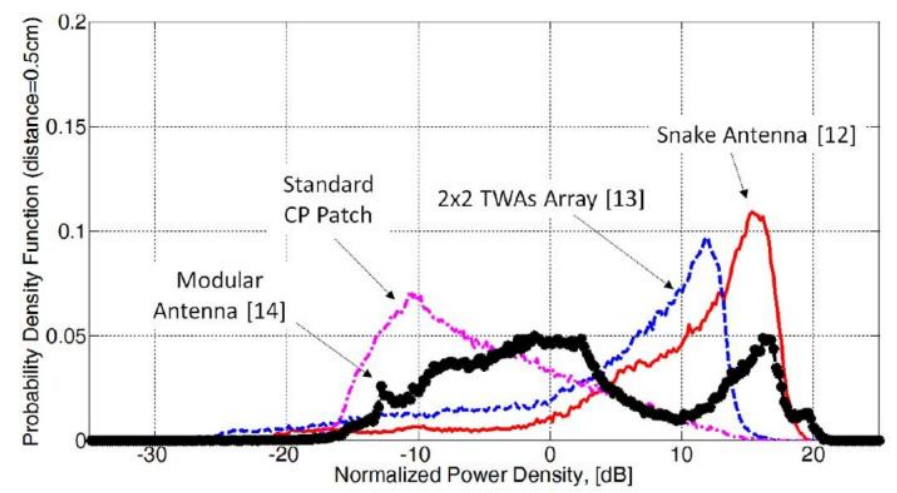

(a)

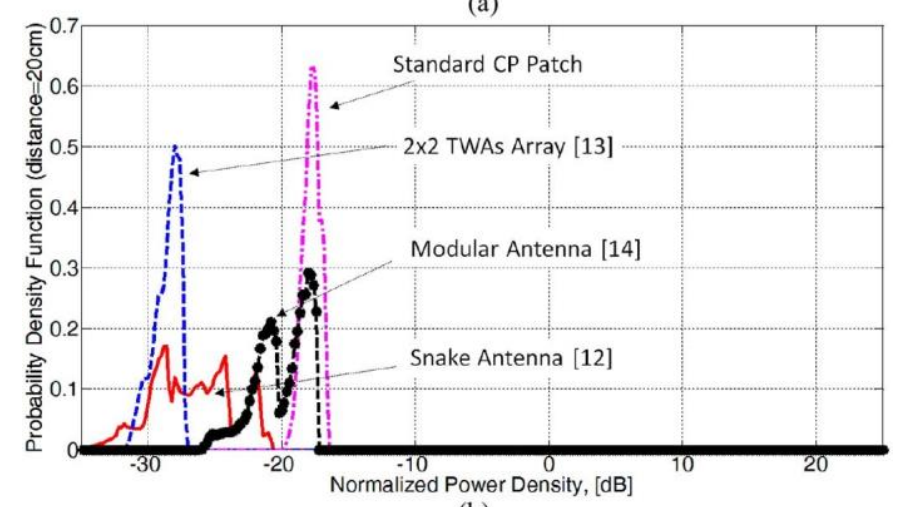

(b)

Fig. 2. Probability density function of the normalized power density computed in an area of $275 \times 136 \mathrm{~mm}^{2}$ for [12]-[14] and $160 \times 160 \mathrm{~mm}{ }^{2}$ for the CP patch, at (a) 0.5 and (b) $20 \mathrm{~cm}$ from the antenna surface. The curves have been obtained by averaging the values computed at different frequencies in the range between 902 and $928 \mathrm{MHz}$ (with a 2-MHz step).

PDF curves are shown for the above-mentioned four antenna configurations, at 0.5 and $20 \mathrm{~cm}$ from the antenna surface. The curves in Fig. 2(a) have been obtained by evaluating (1) on a grid at $0.5 \mathrm{~cm}$ over the antenna surface. The curve for the $\mathrm{CP}$ patch demonstrates that the field distribution on the surface is not as uniform as for the other configurations. Moreover, as already shown in Fig. 1, the NPD for the patch is on average lower than that related to the other configurations. Furthermore, by increasing the distance from the antenna surface up to $20 \mathrm{~cm}$ [Fig. 2(b)], the NPD distribution tends to become uniform for all the antenna configurations, as expected since the radiated field approaches a spherical wave behavior. For the snake antenna and TWAs array, the average value is lower than the modular antenna and $\mathrm{CP}$ patch ones, which is an expected result as they exhibit a lower far-field gain. 
III.

To verify the effectiveness of the NPD numerical analysis in a qualitative estimate of the performance of an NF UHFRFID reader antenna, tag detection tests have been carried out using all four antenna configurations considered in Section II. Tag detection tests have been done with a commercial UHF RFID reader (CAEN R4300P-Ion), with an input power equal to $200 \mathrm{~mW}$, in the FCC RFID band (902-928 MHz) and enabling the frequency hopping functionality. The tag LAB ID Inlay UH113 $\left(18 \times 32 \mathrm{~mm}^{2}\right)$ has been used, which is a compact-size tag made

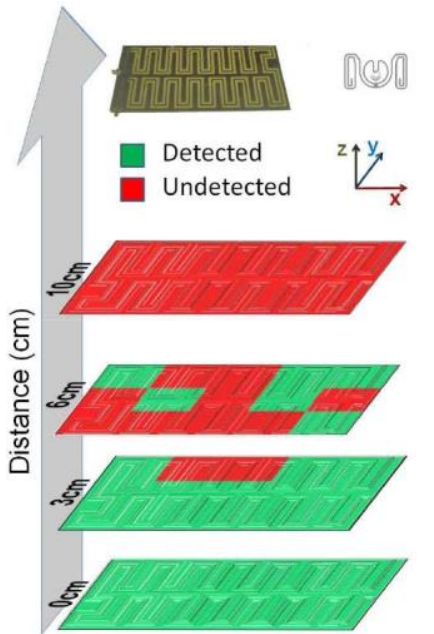

(a)

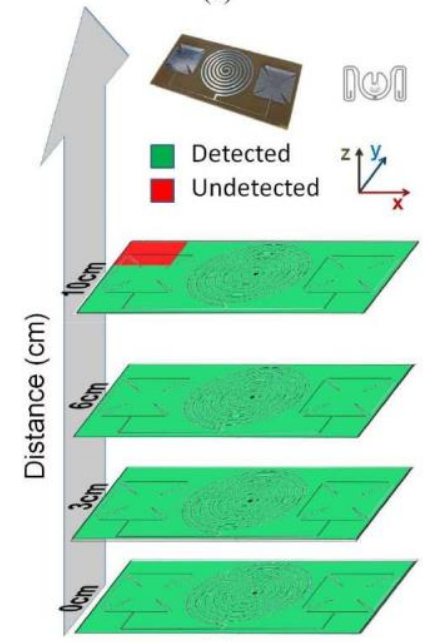

(c)

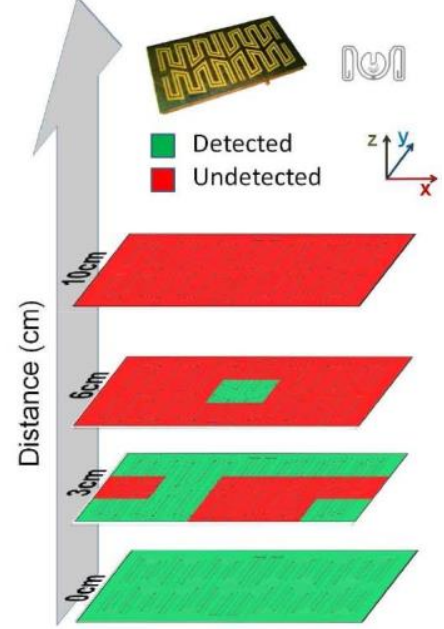

(b)

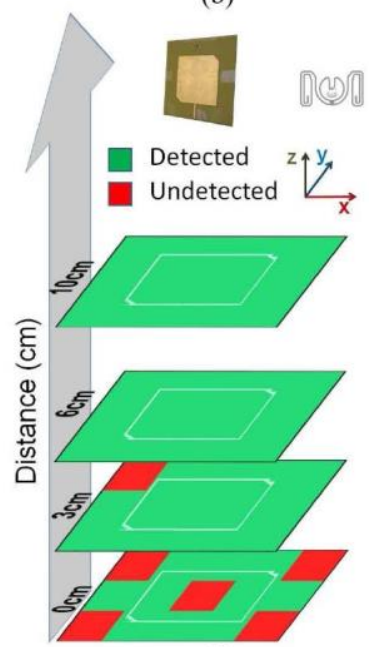

(d)

Fig. 3. Tag detection test for the (a) snake antenna, (b) meandered TWAs array,,modular antenna, and (d) CP patch. A UH113 tag has been used, by varying distance and position of the tag with respect to antenna surface.

of a folded dipole with a loop [16]. Rectangular test surfaces parallel to the antenna have been subdivided into $3 \times 5$ cells. The detection test has been repeated in each cell by varying the distance of the tag from the antenna surface. The results of the detection tests are shown in Fig. 3, by considering a tag-reader antenna distance equal to 0 (the tag is placed directly on the antenna surface), 3,6 , and $10 \mathrm{~cm}$. Measured results confirm the reading performance that was expected on the basis of the NPD numerical analysis shown in Section II. As shown in Fig. 1, the field decay rate is higher for the snake antenna [Fig. 3(a)] and the meandered TWAs array [Fig. 3(b)], so exhibiting a satisfactory tag detection at distances shorter than 3 $\mathrm{cm}$ along with a rapid reading performance degradation above such limit. On the other hand, for the modular antenna [Fig. 3(c)] and CP patch [Fig. 3(d)], the field decay rate is lower (Fig. 1), resulting in satisfactory reading performance up to $10 \mathrm{~cm}$ and beyond. Furthermore, the poor field distribution uniformity shown in Fig. 2 for the CP patch is confirmed by the poor reading performance when the tag is directly placed above the antenna surface [Fig. 3(d)]. Conversely, for the other antennas that have been designed ad hoc for NF desktop readers, a tag placed on the antenna surface can be detected 
regardless of its specific position, as predicted by the corresponding PDF curves in Fig. 2.

In conventional far-field UHF-RFID systems, for a given set of reader antenna and tag, the read range can be estimated through a well-known simple link-budget equation. It is ap- parent that in an NF UHF-RFID system, the NPD cannot be used to analytically calculate the read range for a given RFID chip sensitivity and reader output power. Nonetheless, it has here been shown that the NPD numerical data can be used at least for a qualitative prediction of the expected performance of NF reader antennas, as well as for a comparison procedure among a set of different antenna layouts, as an alternative to either time-consuming experimental reading measurements or full-wave numerical simulations of the electromagnetic system made of both the reader and tag antennas. Although both measurements and full-wave simulations can give more accurate performance estimations, their adoption unavoidably calls for a specific selection of the tag topology. Therefore, the extension of the performance analysis results to other tags, without additional measurements or full-wave simulations, inevitably introduces uncertainties that can be of the same order of those accepted by a procedure based on the proposed NPD numerical analysis.

IV.

\section{CONCLUSION}

The power density (Euclidean norm of the real component of the Poynting vector) has been proposed as a metric to numerically assess the performance of NF UHF-RFID antennas. A proper normalization factor has been included to allow for the performance comparison among reader antennas with different size. The spatial decay rate of the normalized power density gives information on the expected behavior of the reading performance versus the distance from the antenna surface. More- over, its probability density function is suitable to qualitatively estimate the probability of missed readings related to low-field regions on the reader antenna surface. Numerical results have been shown for three antennas presented in the open literature for near-field UHF-RFID applications, as well as for a standard resonant patch antenna. Measurement results through a UHF- RFID setup demonstrated that NPD numerical data can be used for the qualitative prediction of the performance of NF reader antennas, as well as for a comparison procedure among a set of different antenna layouts, regardless on the tag topology. NPD numerical analysis can be a valid alternative to either timeconsuming experimental reading measurements or full-wave numerical simulations of the whole electromagnetic system made of both the reader and tag antennas. Finally, it is worth noting that the proposed power density analysis can be also applied to low-profile near-field UHF-RFID reader antennas to be integrated into shelves, drawers, access- control barriers, etc.

\section{ACKNOWLEDGMENT}

The authors would like to acknowledge CAEN RFID s.r.l. for the technical support and for providing the measurement setup.

\section{REFERENCES}

[1] P. V. Nikitin, K. V. S. Rao, and S. Lazar, “An overview of near field UHF RFID,” in Proc. IEEE Int. Conf. RFID, Mar. 26-28, 2007, pp. $167-174$.

[2] A. Buffi, A. A. Serra, P. Nepa, H.-T. Chou, and G. Manara, "A focused planar microstrip array for 2.4 GHz RFID readers," IEEE Trans. Antennas Propag., vol. 58, no. 5, pp. 1536-1544, May 2010.

[3] A. S. Andrenko, "Optimized near-field antenna for UHF RFID smart shelf applications," in Proc. IEEE Antennas Propag. Symp. APSURSI, Vancouver, BC, Canada, 2015, pp. 1576-1577.

[4] W. Choi, J. S. Kim, J. H. Bae, G. Choi, and J. S. Chae, "Near-field antenna for a radio frequency identification shelf in the UHF band," Microw., Antennas Propag., vol. 4, no. 10, pp. 1538-1542, 2010.

[5] D. M. Dobkin, S. M. Weigand, and N. Iye, "Segmented magnetic antennas for near-field UHF RFID," Microw. J., Jun. 2007.

[6] M. Daiki, E. Perret, and S. Tedjini, "Design of near field UHF RFID reader antenna integrated into clothes," in Proc. IEEE RFID-TA, Tampere, Finland, Sep. 2014, pp. 261-265.

[7] B. Shrestha, A. Elsherbeni, and L. Ukkonen, "UHF RFID reader antenna for near-field and far-field operations," IEEE Antennas Wireless Propag. Lett., vol. 10, pp. 1274-1277, 2011.

[8] J. Shi, X. Qing, and Z. N. Chen, "Electrically large zero-phase-shift line grid-array UHF near-field RFID reader antenna," IEEE Trans. Antennas Propag., vol. 62, no. 4, pp. 2201-2208, Apr. 2014.

[9] C. R. Medeiros, J. R. Costa, and C. A. Fernandes, "RFID reader antennas for tag detection in self-confined volumes at UHF," IEEE Antennas Propag. Mag., vol. 53, no. 2, pp. 39-50, Apr. 2011.

[10] W. S. Lee, K. S. Oh, and J. W. Yu, "Design of spiral-shaped UHF near-field reader antenna for RFID applications," in Proc. IEEE MTT-S IMWS-IRFPT, 2011, pp. 1-2.

[11] A. Ren, C. Wu, Y. Gao, and Y. Yuan, “A robust UHF near-field RFID reader antenna,” IEEE Trans. Antennas Propag., vol. 60, no. 4, pp. 1690-1697, Apr. 2012.

[12] A. Michel et al., "Design and performance analysis of a planar antenna for near-field UHFRFID desktop readers," in Proc. APMC, Kaohsiung, Taiwan, Dec. 2012, pp. 1019-1021. 
[13] A. Michel, R. Caso, A. Buffi, P. Nepa, and G. Isola, "Meandered TWAS array for near-field UHF RFID applications," Electron. Lett., vol. 50, no. 1, pp. 17-18, Jan. 2, 2014.

[14] R. Caso, A. Michel, A. Buffi, P. Nepa, and G. Isola, "A modular antenna for UHF RFID near-field desktop reader," in Proc. IEEE RFIDTA, Tampere, Finland, Sep. 2014, pp. 204-207.

[15] A. Michel, R. Caso, A. Buffi, P. Nepa, and G. Isola, "Modular antenna for reactive and radiative near-field regions of UHF-RFID desktop readers," in Proc. 31st URSI GASS, Beijing, China, Aug. 2014, pp. 1-4.

[16] LAB ID, "Inlay UHF-UH113,” 2011 [Online]. Available: http://www.lab-id.com/datasheet/inlay_UHF/UH113.pdf 